Daniela Ardalić, Aleksandra Stefanović*, Jelena Kotur-Stevuljević, Ana Ninić, Slavica Spasić, Vesna Spasojević-Kalimanovska, Zorana Jelić-Ivanović and Željko Miković

\title{
Lipid indexes and parameters of lipid peroxidation during physiological pregnancy
}

https://doi.org/10.1515/labmed-2018-0026

Received March 7, 2018; accepted December 30, 2018; previously published online March 22, 2019

\section{Abstract}

Background: Specific metabolic changes during physiological pregnancy are characterized by hyperlipidemia and increased oxidative stress. However, these specific changes raise the question of their pro-atherogenic potential during pregnancy and their influence on the risk of developing cardiovascular disease (CVD) in women later in life, as well as complications during pregnancy. The aim of this study was to investigate the changes in lipid indexes and parameters of lipid peroxidation in non-complicated pregnancy in order to estimate their course of change and potential relationship during non-complicated pregnancy. Methods: The study included 43 healthy pregnant women and 38 non-pregnant healthy women, in appropriate ages, as the control group. Lipid parameters and oxidative stress parameters were monitored in a longitudinal study in the first, second and third trimesters, and before delivery during non-complicated pregnancy.

Results: Results have shown that lipid indexes rise during pregnancy. The values were significantly increased when compared to the first trimester in all lipid indices and in comparison with the control group. Thyobarbituric acid reactive substances (TBARS) and lipid hydroperoxides (LOOH) were not changed significantly during physiological pregnancy, but LOOH showed a significantly higher value in the first trimester compared with the control group. Prooxidative-antioxidative balance (PAB) significantly increases as pregnancy progresses.

Conclusions: We observed the changes in lipids, lipid indexes and parameters that indicate oxidative

*Correspondence: Prof. Aleksandra Stefanović, Department of Medical Biochemistry, Faculty of Pharmacy, University of Belgrade, Vojvode Stepe 450, Belgrade, Serbia, E-Mail: alex@pharmacy.bg.ac.rs Daniela Ardalić and Željko Miković: Clinic of Gynecology and Obstetrics, Belgrade, Serbia Jelena Kotur-Stevuljević, Ana Ninić, Slavica Spasić, Vesna Spasojević-Kalimanovska and Zorana Jelić-Ivanović: Department of Medical Biochemistry, Faculty of Pharmacy, University of Belgrade, Belgrade, Serbia modification of lipids in physiological pregnancy that may lead to an atherogenic, prooxidant state.

Keywords: hyperlipidemia; lipid indexes; lipid peroxidation; pregnancy.

\section{Introduction}

Non-complicated (physiological) pregnancy is a dynamic state accompanied by specific metabolic changes. Among these changes, the most interesting for researchers in the last couple of years were specific lipid profile and oxidative stress status, because of their potential influence on women's health later in life and their influence on cardiovascular disease (CVD) development [1-3]. In addition, these alterations in lipogenesis and oxidative stress status have been linked to perinatal morbidity and mortality, as a popular area for research outcomes [3-7]. Specific altered lipid profile during non-complicated pregnancy is essential for the normal course of pregnancy and fetal development. Nevertheless, these specific changes in lipid parameters raise the question of their pro-atherogenic potential during pregnancy and its influence on the risk for the development of CVD in women later in life, as well as complications during pregnancy, especially preeclampsia, but also gestational diabetes mellitus and intrauterine growth restriction (IUGR). By the end of the third trimester, most healthy pregnant women develop a lipid profile that could be considered highly atherogenic in healthy nonpregnant women [1]. Non-complicated pregnancy is also associated with alterations in the composition and size of low-density lipoprotein (LDL) and high-density lipoprotein (HDL) particles, which become smaller and denser with higher pro-atherogenic potential and decreased atheroprotective potential [6, 7]. Apolipoprotein A-I (apoAI) and apolipoprotein $B(a p o B)$ are considered to be better indicators of pro-atherogenic and atheroprotective lipid components, because of their lower metabolic variations compared to other lipid components [8]. ApoB is an essential structural component of very low-density lipoproteins (VLDLs), intermediate-density lipoproteins (IDLs) and LDL. As each particle in these lipoproteins contains 
apoB, the total number of atherogenic particles can be estimated by measuring the plasma level of this apolipoprotein. The levels of apoA-I in plasma are strongly correlated with HDL-cholesterol (HDL-C) levels and generally with HDL particles with confirmed antiatherogenic effects $[9,10]$. The ratio apoB/apoA-I is considered to be the best indicator of the pro-atherogenic and atheroprotective components of lipoprotein particles [8]. Similarly, the atherogenic index of plasma (AIP) is considered a good indicator for the presence of small dense particles [8], so the combination of these indexes provides good information about the characteristics of dyslipidemia.

Non-complicated pregnancy is also characterized by increased oxidative stress. Reactive oxygen species (ROS) and their control by antioxidants are involved in the physiology of the female reproductive system [2]. They are important for the normal course of pregnancy and fetal development. When the balance with the antioxidant system is disturbed, oxidative stress in pregnancy may lead to serious complications, such as preeclampsia, gestational diabetes mellitus, IUGR, miscarriage and preterm birth $[2,11]$. Increase in oxidative stress is associated with abnormal lipid profile and may cause oxidative modification of lipids, so the studies which were conducted in complicated pregnancies also showed increased concentrations of lipid peroxides [2, 12, 13]. Altered lipid profile, oxidative stress and inflammation are molecular mediators of endothelial dysfunction development, which leads to preeclampsia and other pregnancy complications [10, $12,14]$.

The aim of this study was to investigate serum lipid profile by observing the changes in the ratios between pro-atherogenic and atheroprotective lipid components expressed as lipid indexes, as well as AIP in non-complicated pregnancy. Also, we wanted to evaluate the changes in markers of oxidative stress and markers of lipid oxidative modifications during non-complicated pregnancy because of their crucial role in the development of pregnancy complications.

\section{Materials and methods}

For the purpose of a longitudinal study, 60 healthy pregnant Caucasian women were recruited during their regular gynecological check-ups at the Gynecology and Obstetrics Clinic "Narodni Front" in Belgrade, Serbia. The study lasted for 2 years; the blood was sampled toward the end of each trimester and before delivery. A full medical history was taken, including noting the presence of any systemic disorders before pregnancy, smoking status, alcohol intake, antioxidant supplementation, and family history of CVD and diabetes mellitus. The median age of the subjects was 26 years (range $20-35$ ). The study protocol also included height and weight measurement for body mass index calculation $(\mathrm{BMI}=$ weight $[\mathrm{kg}] /$ squared height $\left.\left[\mathrm{m}^{2}\right]\right)$. Exclusion criteria from the study were overweight before pregnancy, as well as the presence of any chronic disease before pregnancy or intake of regular medication affecting the lipid profile and oxidative stress status, multiple gestation, molar pregnancy, gestational hypertension, gestational diabetes, preeclampsia, the presence of any other complications in pregnancy and preterm delivery. Based on these criteria, 17 pregnant women were excluded from the study, so finally data from 43 pregnant women were fully processed. The control group consisted of 38 healthy women. Exclusion criteria for controls were inflammation, infection, presence of neurological dysfunction, and a history of CVD, renal, hepatic, gastrointestinal, pulmonary, endocrine or oncological disease. The control and pregnancy groups were matched for age. Responses to a standard questionnaire were collected in person by trained interviewers. The whole study was planned according to the ethical standards detailed in the Declaration of Helsinki (as revised in 1983) and according to local institutional guidelines.

Venous blood was drawn from each subject's antecubital vein after nighttime fasting (12 h) into one serum sample tube and one ethylenediaminetetraacetic acid (EDTA) sample tube before centrifugation at $1500 \times g$ for $10 \mathrm{~min}$. Plasma and serum samples were stored at $-80^{\circ} \mathrm{C}$ in aliquots until analysis (1 month after the blood sampling).

Basic biochemical parameters were determined using an Ilab 300 Plus auto-analyzer employing commercial kits (Bioanalytica, Belgrade, Serbia). Hematology parameters were determined using the hematological analyzer Cel-Dyn 1700 (Abbott Diagnostics, Chicago, IL, USA). AIP was calculated according to the following equation: AIP $=\log$ (triglyceride [TG]/HDL-C), with units for TG and HDL-C in $\mathrm{mmol} / \mathrm{L}$ [14]. The other lipid indexes were expressed through simple ratios of lipid components: apoB/apoA-I, cholesterol (CHOL)/HDL-C, LDL-C/HDL-C and TG/HDL-C. The concentration of thyobarbituric acid-reactive substances (TBARS) was measured using the molar absorption coefficient of $1.56 \times 105 \mathrm{M}^{-1}$ at $535 \mathrm{~nm}$, as described by Girotti et al. [15]. Concentrations of lipid hydroperoxides (LOOH) were measured in serum by the ferric-xylenol orange method [16]. The prooxidant-antioxidant balance (PAB) was measured using a method described by Alamdari et al. [17], in which 3,3',5,5'-tetramethyl benzidyne 
and its cation are used as redox indicators participating in two simultaneous reactions.

\section{Statistical analysis}

The assessment of the normality of data distribution was done using the Kolmogorov-Smirnov test. Data were shown as the mean value and standard deviation for normally distributed variables and as the geometric mean and the $95^{\text {th }}$ confidence intervals derived from log-normal values. The comparisons of two groups of continuous variables were performed using Student's t-test. A comparison of continuous variables (more than two groups) was performed using the analysis of variance (repeated measures) with post hoc Bonferroni correction. All statistical analyses were performed using the MedCalc Software (Ostend, Belgium). All statistical tests were considered significant at the probability level of 0.05 .

\section{Results}

General biochemical and hematological parameters are shown in Table 1.

As was expected, there was no difference in blood pressure between the investigated groups. All observed changes in biochemical and hematological parameters which were statistically significant between the first trimester and late pregnancy, as well as between the control group of non-pregnant women and the first trimester of pregnancy, were expected (Table 1).

In Table 2, we present the term of delivery and general characteristics of newborns. All 43 pregnancies ran their normal course of pregnancy and outcome, and delivery at expected terms. Also, all babies born were healthy with a high Apgar score (>8) (Table 2).

Total CHOL, TG, LDL-C and HDL-C rose significantly as the pregnancies progressed in comparison with the first trimester (Table 3).

Table 1: Biochemical and hematological parameters during physiological pregnancy.

\begin{tabular}{|c|c|c|c|c|c|}
\hline Parameter & First trimester & Second trimester & Third trimester & $38^{\text {th }}$ Week of gestation & Control group \\
\hline Week of gestation & $12.9 \pm 0.65$ & $23.2 \pm 0.52$ & $31.7 \pm 0.12$ & $37.3 \pm 0.12$ & - \\
\hline $\mathrm{SBP}, \mathrm{mmHg}$ & $111.7 \pm 11.8$ & $109.5 \pm 11.0$ & $108.3 \pm 8.6$ & $107.8 \pm 9.8$ & $118.3 \pm 11.8$ \\
\hline $\mathrm{DBP}, \mathrm{mmHg}$ & $71.3 \pm 8.7$ & $70.2 \pm 7.4$ & $69.4 \pm 6.5$ & $68.6 \pm 7.5$ & $76.3 \pm 10.6$ \\
\hline Weight gain, \% & $4.2 \pm 4.10$ & $13.6 \pm 5.37^{\mathrm{b}, \mathrm{f}}$ & $21.6 \pm 6.47^{\mathrm{b}, \mathrm{g}, \mathrm{c}, \mathrm{f}}$ & $25.2 \pm 7.92^{\mathrm{b}, \mathrm{g}, \mathrm{g}, \mathrm{f}, \mathrm{d}, \mathrm{f}, \mathrm{f}}$ & - \\
\hline WBC, $\times 10^{9}$ & $8.1 \pm 1.96$ & $9.3 \pm 2.05^{b, f}$ & $9.7 \pm 2.21^{\mathrm{b}, \mathrm{f}}$ & $10.2 \pm 5.40^{b, f}$ & $5.7 \pm 1.29^{\mathrm{b}, \mathrm{h}, \mathrm{c}, \mathrm{i}, \mathrm{d}, \mathrm{i}, \mathrm{e}, \mathrm{i}}$ \\
\hline $\mathrm{RBC}, \times 10^{12}$ & $4.0 \pm 0.23$ & $3.7 \pm 0.25$ & $3.8 \pm 0.25$ & $3.9 \pm 0.28$ & $4.42 \pm 0.32$ \\
\hline Hemoglobin, $\mathrm{g} / \mathrm{L}$ & $124.5 \pm 8.49$ & $115.8 \pm 8.37$ & $115.0 \pm 8.28$ & $118.9 \pm 9.42$ & $131.0 \pm 8.29$ \\
\hline Hematocrit, \% & $35.6 \pm 2.28$ & $33.0 \pm 2.30$ & $33.5 \pm 2.27$ & $34.5 \pm 2.57$ & $39.8 \pm 2.83$ \\
\hline $\mathrm{MCH}, \mathrm{pg}$ & $30.7 \pm 1.36$ & $31.0 \pm 1.41$ & $30.3 \pm 1.72^{c, f}$ & $30.2 \pm 2.01^{c, f}$ & $29.7 \pm 1.69^{\mathrm{b}, \mathrm{i}, \mathrm{c}, \mathrm{i}}$ \\
\hline Platelets, $\times 10^{9}$ & $217.7 \pm 50.52$ & $236.9 \pm 54.18$ & $218.8 \pm 50.78$ & $216.0 \pm 56.66$ & $210.5 \pm 48.82$ \\
\hline Total protein, $\mathrm{g} / \mathrm{L}$ & $68.7 \pm 10.16$ & $64.2 \pm 6.13$ & $63.8 \pm 3.20^{\mathrm{b}, \mathrm{f}}$ & $63.7 \pm 4.34^{\mathrm{b}, \mathrm{f}}$ & $70.5 \pm 3.73^{c, i, d, i, e, i}$ \\
\hline Glucose, $\mathrm{mmol} / \mathrm{L}$ & $4.3 \pm 0.44$ & $4.3 \pm 0.48$ & $4.5 \pm 0.50$ & $4.3 \pm 0.48$ & $4.4 \pm 0.45$ \\
\hline Urea, $\mathrm{mmol} / \mathrm{L}$ & $3.1 \pm 0.68$ & $2.9 \pm 0.75$ & $2.6 \pm 0.84^{b, f}$ & $2.7 \pm 0.77^{b, f}$ & $4.4 \pm 1.09^{\mathrm{b}, \mathrm{i}, \mathrm{c}, \mathrm{i}, \mathrm{d}, \mathrm{i}, \mathrm{e}, \mathrm{i}}$ \\
\hline Creatinine, $\mu \mathrm{mol} / \mathrm{L}$ & $63.6 \pm 6.41$ & $62.4 \pm 6.51$ & $62.9 \pm 8.03$ & $67.9 \pm 7.61^{b, g, c, g, g, d, g}$ & $63.5 \pm 8.41^{\mathrm{e}, \mathrm{i}}$ \\
\hline AST, U/L & $17.5 \pm 2.97$ & $20.2 \pm 7.74$ & $21.7 \pm 7.49^{b, f}$ & $23.0 \pm 12.71^{b, f}$ & $18.2 \pm 2.55$ \\
\hline ALT, U/L & $14.4 \pm 6.17$ & $19.4 \pm 17.00$ & $21.9 \pm 21.28$ & $22.5 \pm 35.18$ & $17.4 \pm 3.12$ \\
\hline
\end{tabular}

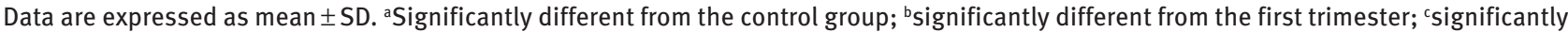
different from the second trimester; ${ }^{\mathrm{d}}$ significantly different from the third trimester; ${ }^{\text {e }}$ significantly different from the $38^{\text {th }}$ week of gestation. ${ }^{f} p<0.05,{ }^{g} p<0.001$ (Tukey's post hoc test), ${ }^{h} p<0.05$ (Student's t-test), ${ }^{i} p<0.001$ (Student's t-test). ALT, alanine aminotransferase; AST, aspartate aminotransferase; DBP, diastolic blood pressure; $\mathrm{MCH}$, mean corpuscular hemoglobin; RBC, red blood cell; SBP, systolic blood pressure; WBC, white blood cell.

Table 2: Term of delivery and newborn characteristics (expressed as mean value \pm SD).

\begin{tabular}{lrrrrr}
\hline $\begin{array}{l}\text { Week of gestation } \\
\text { at delivery term }\end{array}$ & $\begin{array}{r}\text { Gender of } \\
\text { newborn } \\
\text { babies } \mathbf{M} / \mathbf{F}\end{array}$ & $\begin{array}{r}\text { The weight } \\
\text { of newborn } \\
\text { babies, } \mathbf{~}\end{array}$ & $\begin{array}{r}\text { The length } \\
\text { of newborn } \\
\text { babies, } \mathbf{c m}\end{array}$ & $\begin{array}{r}\text { The head circumference } \\
\text { of newborn babies, } \mathbf{c m}\end{array}$ & $\begin{array}{r}\text { Apgar score } \\
\text { of newborn babies } \\
\text { at } \mathbf{1} \text { and } \mathbf{5} \text { min }\end{array}$ \\
\hline $39.5 \pm 1.00$ & $22 / 21$ & $3459.3 \pm 426.26$ & $51.6 \pm 2.04$ & $35.3 \pm 1.03$ & $8.9 \pm 1.11 / 9.7 \pm 0.98$ \\
\hline
\end{tabular}

SD, standard deviation. 
Table 3: Lipid parameters and lipid indexes during physiological pregnancy.

\begin{tabular}{|c|c|c|c|c|c|}
\hline Lipid and lipid indices & First trimester & Second trimester & Third trimester & $38^{\text {th }}$ Week of gestation & Control group \\
\hline $\mathrm{CHOL}, \mathrm{mmol} / \mathrm{L}$ & $4.9 \pm 0.79$ & $6.3 \pm 0.97^{\mathrm{b}, \mathrm{g}}$ & $6.8 \pm 1.05^{\mathrm{b}, \mathrm{g}, \mathrm{c}, \mathrm{g}}$ & $6.9 \pm 1.10^{\mathrm{b}, \mathrm{g}, \mathrm{c}, \mathrm{g}}$ & $4.5 \pm 0.71^{b, i, c, i, d, i, e, i}$ \\
\hline $\mathrm{TG}, \mathrm{mmol} / \mathrm{L}^{\mathrm{a}}$ & $1.0(0.95-1.12)$ & $1.6^{\mathrm{b}, \mathrm{g}}(1.49-1.79)$ & $2.3^{g, c, g}(2.14-2.56)$ & $2.7^{\mathrm{b}, \mathrm{g}, \mathrm{c}, \mathrm{g}, \mathrm{d}, \mathrm{g}}(2.54-3.01)$ & $0.8^{\mathrm{b}, \mathrm{i}, \mathrm{c}, \mathrm{i}, \mathrm{d}, \mathrm{i}, \mathrm{e}, \mathrm{i}}(0.71-0.90)$ \\
\hline $\mathrm{HDL}-\mathrm{C}, \mathrm{mmol} / \mathrm{L}$ & $1.9 \pm 0.36$ & $2.3 \pm 0.50^{\mathrm{b}, \mathrm{g}}$ & $2.1 \pm 0.52^{\mathrm{c}, \mathrm{g}}$ & $1.9 \pm 0.45^{c, g, d, f}$ & $1.5 \pm 0.30^{\mathrm{b}, \mathrm{i}, \mathrm{c}, \mathrm{i}, \mathrm{i}, \mathrm{d}, \mathrm{i}, \mathrm{e}, \mathrm{i}}$ \\
\hline LDL-C, mmol/L & $2.5 \pm 0.73$ & $3.2 \pm 0.96^{\mathrm{b}, \mathrm{g}}$ & $3.7 \pm 1.04^{\mathrm{b}, \mathrm{g}, \mathrm{c}, \mathrm{g}}$ & $3.6 \pm 1.08^{\mathrm{b}, \mathrm{g}, \mathrm{c}, \mathrm{f}}$ & $2.6 \pm 0.66^{\mathrm{c} \text { ci, d,i,e,h }}$ \\
\hline Apo-Al, g/L & $1.5 \pm 0.29$ & $1.7 \pm 0.28^{\mathrm{b}, \mathrm{g}}$ & $1.7 \pm 0.32^{\mathrm{b}, \mathrm{g}}$ & $1.6 \pm 0.30^{\mathrm{b}, \mathrm{f}}$ & $1.5 \pm 0.18^{c, i, d, i, e, h}$ \\
\hline ApoB, g/L & $0.6 \pm 0.14$ & $0.8 \pm 0.15^{\mathrm{b}, \mathrm{g}}$ & $1.0 \pm 0.17^{\mathrm{b}, \mathrm{g}, \mathrm{c}, \mathrm{g}}$ & $1.1 \pm 0.18^{\mathrm{bf,g,c,g, \textrm {d } , \mathrm { g }}}$ & $0.8 \pm 0.15^{\mathrm{b}, \mathrm{i}, \mathrm{d}, \mathrm{i}, \mathrm{e}, \mathrm{i}}$ \\
\hline ApoB/ApoA-I & $0.44 \pm 0.15$ & $0.50 \pm 0.14^{\mathrm{b}, \mathrm{f}}$ & $0.63 \pm 0.22^{\mathrm{b}, \mathrm{g}, \mathrm{c}, \mathrm{g}}$ & $0.70 \pm 0.21^{\mathrm{b}, \mathrm{g}, \mathrm{c}, \mathrm{g}, \mathrm{d}, \mathrm{f}}$ & $0.50 \pm 0.13^{\mathrm{a}, \mathrm{h}, \mathrm{c}, \mathrm{i}, \mathrm{d}, \mathrm{i}}$ \\
\hline $\mathrm{CHOL} / \mathrm{HDL}-\mathrm{C}$ & $2.6 \pm 0.64$ & $2.9 \pm 0.74^{b, f}$ & $3.5 \pm 1.19^{\mathrm{b}, \mathrm{g}, \mathrm{c}, \mathrm{g}}$ & $3.8 \pm 1.12^{\mathrm{b}, \mathrm{g}, \mathrm{c}, \mathrm{g}, \mathrm{d}, \mathrm{f}, \mathrm{f}}$ & $3.1 \pm 0.77^{\mathrm{d}, \mathrm{i}, \mathrm{e}, \mathrm{i}}$ \\
\hline LDL-C/HDL-C & $1.3 \pm 0.56$ & $1.5 \pm 0.67$ & $2.0 \pm 0.99^{b, g, c, g}$ & $2.0 \pm 0.91^{\mathrm{b}, \mathrm{g}, \mathrm{g}, \mathrm{g}}$ & $1.8 \pm 0.66^{\mathrm{b}, \mathrm{i}, \mathrm{c}, \mathrm{h}}$ \\
\hline TG/HDL-C & $0.6 \pm 0.24$ & $0.8 \pm 0.27^{\mathrm{b}, \mathrm{g}}$ & $1.3 \pm 0.59^{\mathrm{b}, \mathrm{g}, \mathrm{c}, \mathrm{g}}$ & $1.6 \pm 0.72^{\mathrm{b}, \mathrm{g}, \mathrm{c}, \mathrm{g}, \mathrm{d}, \mathrm{dg}}$ & $0.6 \pm 0.46^{\mathrm{c}, \mathrm{i}, \mathrm{d}, \mathrm{i}, \mathrm{e}, \mathrm{i}}$ \\
\hline AIP & $-0.3 \pm 0.16$ & $-0.1 \pm 0.17^{\mathrm{b}, \mathrm{g}}$ & $0.1 \pm 0.20^{b, g, c, g}$ & $0.2 \pm 0.19^{\mathrm{b}, \mathrm{g}, \mathrm{g}, \mathrm{g}, \mathrm{d}, \mathrm{g}}$ & $-0.3 \pm 0.23^{c, i, \mathrm{~d}, \mathrm{~h}, \mathrm{e}, \mathrm{i}}$ \\
\hline
\end{tabular}

Data are expressed as mean $\pm S D$; ${ }^{\text {means }}$ and $95^{\text {th }}$ confidence intervals derived from log-normal values. ${ }^{b}$ Significantly different from $1^{\text {st }}$

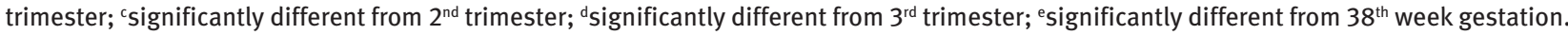
${ }^{f} \mathrm{p}<0.05,{ }^{\mathrm{g}} \mathrm{p}<0.001$ (Tukey's post hoc test). ${ }^{\mathrm{h}} \mathrm{p}<0.05$ (Student's t-test), $\mathrm{p} p<0.001$ (Student's t-test). apoA-I, apolipoprotein A-I; apoB, apolipoprotein B; CHOL, cholesterol; HDL-C, high-density lipoprotein-cholesterol; LDL-C, low-density lipoprotein-cholesterol; TG, trigylcerides.

HDL-C showed the highest value in the second trimester, but by the end of pregnancy this value declined and in the $38^{\text {th }}$ week of pregnancy it was significantly lower than in the second trimester (Table 3). Lipid indexes that showed relations among lipid parameter growth during pregnancy indicated an increase in pro-atherogenic components of lipid parameters. The values increased significantly when compared to the first trimester in all the lipid indexes. CHOL/HDL-C and LDL-C/HDL-C indexes were significantly lower in the first trimester than in the control group due to an increase in HDL-C and LDL-C compared to total CHOL. As pregnancy progressed, these indices grew and were significantly higher than in the first trimester (Table 3).

ApoB/apoA-I and AIP lipid indexes also increased significantly during physiological pregnancy, indicating an increase in pro-atherogenic components as pregnancy progresses. TBARS is the final product of decomposition of lipid peroxides and represents a late-stage marker of oxidant damage. During pregnancy, these values are mostly unchanged, and statistical analysis showed that there was no significant difference in the values during pregnancy compared to the start of pregnancy and compared to non-pregnant women (Table 4).

The movement in the values of $\mathrm{LOOH}$ during pregnancy was similar, but the values at the beginning of pregnancy were significantly higher than the values in the control group.

PAB showed significantly higher values in the first trimester of pregnancy compared to the values of the control group of non-pregnant women, and also in all the examined periods of pregnancy compared to the first trimester (Table 4).

\section{Discussion}

Pregnancy in healthy women is associated with physiological hyperlipidemia that is essential for the normal course of pregnancy and fetal growth and development. However, recent studies have shown that a remarkably altered lipid profile during pregnancy is associated with

Table 4: Oxidative stress parameters during physiological pregnancy.

\begin{tabular}{|c|c|c|c|c|c|}
\hline Parameter & First trimester & Second trimester & Third trimester & $38^{\text {th }}$ Week of gestation & Control group \\
\hline TBARS, $\mu \mathrm{mol} / \mathrm{L}$ & $1.0 \pm 0.28$ & $1.1 \pm 0.30$ & $1.1 \pm 0.35$ & $1.0 \pm 0.22$ & $0.9 \pm 0.42$ \\
\hline LOOH, $\mu \mathrm{mol} / \mathrm{L}$ & $9.5 \pm 2.1$ & $9.5 \pm 1.80$ & $9.2 \pm 2.39$ & $9.4 \pm 2.57$ & $7.9 \pm 1.84^{b, h, c, h, d, h, e, h}$ \\
\hline PAB, HK units ${ }^{a}$ & $\begin{array}{r}107.1 \\
(102.9-123.3)\end{array}$ & $\begin{array}{r}146.0^{\mathrm{b}, \mathrm{g}} \\
(142.5-154.2)\end{array}$ & $\begin{array}{r}160.6^{\mathrm{b}, \mathrm{g}, \mathrm{c}, \mathrm{g}} \\
(147.4-170.2)\end{array}$ & $\begin{array}{r}170.7^{b, g, c, g, d, f} \\
(155.4-185.3)\end{array}$ & $\begin{array}{r}68.6^{\mathrm{b}, \mathrm{i}, \mathrm{c}, \mathrm{i}, \mathrm{d}, \mathrm{i}, \mathrm{e}, \mathrm{i}} \\
(61.4-76.6)\end{array}$ \\
\hline
\end{tabular}

Data are expressed as mean \pm SD; a means and the $95^{\text {th }}$ confidence intervals derived from log-normal values. ${ }^{\text {b }}$ Significantly different from the first trimester; 'significantly different from the second trimester; ${ }^{\mathrm{d}}$ significantly different from the third trimester; ${ }^{\mathrm{e}}$ significantly different from the $38^{\text {th }}$ week of gestation. ${ }^{\mathrm{f}} \mathrm{p}<0.05,{ }^{\mathrm{g}} \mathrm{p}<0.001$ (Tukey's post hoc test). ${ }^{\mathrm{h}} \mathrm{p}<0.05$ (Student's t-test), $\mathrm{i} p<0.001$ (Student's t-test). LOOH, lipid hydroperoxides; PAB, prooxidative-antioxidative balance; TBARS, thyobarbituric acid reactive substances. 
perinatal morbidity and mortality, so dyslipidemia in pregnancy is placed in the focus of actual investigations [3]. Results of the current study have shown that during non-complicated pregnancy, as it progresses, there is an increase in pro-atherogenic components of the lipid profile (CHOL, TG, LDL-C and apoB), and they reach maximum values in the third trimester (Table 3). These results are in agreement with previous studies which indicate that physiological pregnancy is associated with an increase in lipid components as pregnancy progresses [1, $18,19]$. During the second trimester of non-complicated pregnancy, there is an increase in HDL-C concentrations and this increase, with a slight decline, is maintained to the end of delivery time $[1,7,18]$. The results of the current study have shown the same characteristics in HDL-C alterations during physiological pregnancy (Table 3). This increase in atheroprotective HDL-C concentrations during non-complicated pregnancy could be explained as a compensatory mechanism in specific physiological dyslipidemias of pregnancy.

Because of specific alterations in lipid profile markers during non-complicated pregnancy (increase in both pro-atherogenic and atheroprotective lipid markers), it is considered that atherogenic indexes are better indicators of lipid profile changes $[1,8,20-22]$. Also, results of previous studies have shown that the values of the atherogenic indexes are significantly higher in pregnancies complicated by preeclampsia and gestational diabetes in comparison with the values in uncomplicated pregnancy $[1,4,6$, 21]. Among them, according to recent research, maybe the most interesting are apoB/apoA-I (due to their low metabolic variations, compared with the CHOL and TG) and AIP. It has been shown that AIP is a good indicator for the presence of small, dense LDL and HDL particles in plasma, and also that apoB/apoA-I is the best indicator of the relationship between pro-atherogenic and atheroprotective lipid components [8]. It is believed that the apoB/apoA-I index is an important indicator of dyslipidemia with the consequent effect of endothelial dysfunction. Any value above 0.8 for women is considered high risk for the development of CVD [8]. AIP in excess of 0.5 has been proposed as the cut-off value, indicating atherogenic risk [1].

Research results in our population of healthy pregnant women have shown a significant increase in these indexes during pregnancy, which indicates an increase in the pro-atherogenic components of the lipid profile as pregnancy progresses and their intensification in the third trimester (Table 3).

Jia et al. [22] in their study stated that the indices CHOL/HDL-C and TG/HDL-C were good indicators of the distribution of HDL-C subclasses, and their simultaneous rise showed the movement of HDL subclasses toward smaller particles, and the weakness of the reverse CHOL transport. Increases in AIP during non-complicated pregnancy (Table 3) also indicate possible changes in LDL particle distribution toward small, dense LDL particles [8]. According to our results, with an increase in the mentioned indices (Table 3), we could speculate that there is a change in the lipoprotein particle composition during non-complicated pregnancy. It is strongly believed that small dense LDL particles contribute to endothelial dysfunction in preeclampsia $[1,6,19]$, so we consider it very important to investigate the distribution of LDL and HDL particles in non-complicated pregnancy and especially in high-risk pregnancies during the first trimester of pregnancy as potential markers for the prediction of preeclampsia development.

There is a great body of evidence that pregnancy is a physiological condition exhibiting a high susceptibility to oxidative stress development [23]. Increased oxidative stress leads to oxidative modifications of lipids, and lipid peroxidation is considered the cause of complications in pregnancy, especially in the development of preeclampsia $[2,11,13,19,20]$. In our study, during physiological pregnancy without complications, the examined parameters of oxidative stress have shown the following characteristics: LOOH and PAB increased significantly in early pregnancy compared to the value in the control group of non-pregnant women (Table 4). As pregnancy advances, the $\mathrm{LOOH}$ values do not change significantly, while $\mathrm{PAB}$ increases significantly during the entire period of pregnancy. TBARS values do not change significantly in pregnant women compared to non-pregnant women, neither at the beginning of pregnancy nor during the course of pregnancy as it advances. Studies have shown that inappropriate antioxidative response of the mother to the generation of ROS, and the lipid peroxidation that occurs during pregnancy, with altered lipid profile, could be the basis of pregnancy complications (preeclampsia, IUGR, fetal death, gestational diabetes, preterm delivery, etc.) [24]. These facts suggest potential importance in the determination of these parameters, especially lipid peroxidation parameters, which are elevated in complicated pregnancies. Additionally, our results have shown that $\mathrm{LOOH}$ and TBARS during physiological pregnancy were stable, so they might be interesting in correlation with the occurrence of complications. The results have also shown that the PAB value increases significantly during pregnancy and is intensified in the third trimester, indicating that pregnancy is a state of continuous oxidative stress which deepens (Table 4). This result is in agreement with previous studies which have shown that oxidative stress parameters increase 
during pregnancy [25]. Taking into consideration the fact that physiological pregnancy is a condition of increased oxidative stress and altered pro-atherogenic lipid profile, it is obvious that these characteristics can lead to the development of atherosclerotic plaques and, consequently, the development of CVD. Similarly, due to an unbalanced and weak adaptive response mechanism during pregnancy, these alterations can lead to complications during pregnancy as confirmed in previous studies [26, 27].

Metabolic changes during physiological non-complicated pregnancy could be a risk factor for the development of CVD later in women's life, as well as for the development of complications in pregnancy. However, these changes during pregnancy in healthy women are under the control of appropriate mechanisms. Accordingly, women with inadequate adaptive response, particularly if they had started pregnancy with inadequate ratios of atheroprotective and pro-atherogenic components, and in the presence of enhanced oxidative modification of lipids, may develop some complications during pregnancy. The findings of the present study suggest a potential role of antioxidant supplementation during pregnancy, especially in subjects with high risk for preeclampsia and IUGR development. Antioxidant use might reduce lipid peroxidation and also improve antioxidant capacity, and possibly prevent or delay the development of pregnancy complications. However, currently available evidence indicates that antioxidative supplementation has little to no effect on relevant outcomes $[28,29]$ and there are no official recommendations for antioxidant supplementation use in pregnancy. However, the importance of lifestyle and dietary habits during pregnancy is beyond question, and the consumption of a varied and balanced diet is essential to ensure favorable outcomes of pregnancy [30].

\section{Limitations}

This study has limitations; first, we used data from a relatively small cohort and further, we did not investigate the underlying mechanisms of dyslipidemia and the development of lipid oxidative modifications in noncomplicated pregnancy. Prospective studies with larger samples are needed.

\section{Conclusions}

In conclusion, we observed the changes in lipids, lipid indexes and oxidative stress parameters during pregnancy that may lead to an atherogenic, prooxidant state. Further, we could speculate that it would be important to determine these parameters in early pregnancy or the first trimester, with the aim of predicting pregnancy complications. Finally, the combination of the investigated lipid indices and parameters of oxidative stress could be a possible biochemical algorithm for the prediction of pregnancy complications.

Author contributions: All the authors have accepted responsibility for the entire content of this submitted manuscript and approved submission.

Research funding: None declared.

Employment or leadership: None declared.

Honorarium: None declared.

Competing interests: The funding organization(s) played no role in the study design; in the collection, analysis, and interpretation of data; in the writing of the report; or in the decision to submit the report for publication.

\section{References}

1. Garduño-Alanís A, Vázquez-de Anda G, Valdés-Ramos R, Talavera JO, Herrera-Villalobos JE, Huitrón-Bravo GG, et al. Predictors of hyperlipidemia during the first half of pregnancy in Mexican women. Nutr Hosp 2015;31:508-13.

2. Al-Gubory K, Fowler PA, Garrel C. The roles of cellular reactive oxygen species, oxidative stress and antioxidants in pregnancy outcomes. Cell Biol 2010;42:1634-50.

3. Wild R, Weedin EA, Wilson D. Dyslipidemia in pregnancy. Cardiol Clin 2015;33:209-15.

4. Kharb S, Nanda S, Batra A. Relationship between cord blood atherogenic index and birth weight in preeclampsia. Mediter J Nutr Metab 2013;6:59-61.

5. Jammalamadaga VS, Abraham P, Sivaprasad P. ApoB/ApoA-1 ratio and nitric oxide levels in pregnancy induced hypertensive women. Int J Res Med Sci 2016;4:1329-34.

6. Ogura K, Miyatake T, Fukui O, Nakamura T, Kameda T, Yoshino G. Low-density lipoprotein particle diameter in normal pregnancy and preeclampsia. J Atheroscler Thromb 2002;9:42-7.

7. Zeljković A, Vekić J, Spasić S, Jelić-Ivanović Z, SpasojevićKalimanovska V, Gojković T, et al. Changes in LDL and HDL subclasses in normal pregnancy and associations with birth weight, birth length and head circumference. Matern Child Health J 2013;17:556-65.

8. Kaneva AM, Potolitsyna NN, Bojko ER, Odland J. The Apolipoprotein B/Apolipoprotein A-I ratio as a potential marker of plasma atherogenicity. Dis Markers 2015;2015:591454.

9. Kim Yj, Park H, Lee HY, Ahn Y, Ha EH, Suh Sh, et al. Paraoxonase gene polymorphism, serum lipid, and oxidized low-density lipoprotein in preeclampsia. Eur J Obstet Gynecol 2007;133:47-52.

10. Saarelainen H, Laitinen T, Raitakari OT, Juonala M, Heiskanen $\mathrm{N}$, Lyyra-Laitinen T, et al. Pregnancy-related hyperlipidemia and endothelial function in healthy women. Circ J 2006;70:768-72. 
11. Macekova D, Kovac G, Hinst J, Illek B, Pereckova J, Baraskova Z, et al. Lipid peroxidation and biochemical parameters in maternal pre-delivery and post-delivery plasma. Biologia 2010;65:170-4.

12. Clausen T, Đurovic S, Henriksen T. Dyslipidemia in early second trimester is mainly a feature of women with early onset preeclampsia. Br J Obste Gyneacol 2001;108:1081-7.

13. Mohanty S, Nayak N, Nanda NN, Rao P. Serum lipids and malondialdehyde levels in primiparous patients with pregnancy induced hypertension. Ind J Clin Biochem 2006;21:189-92.

14. Sánchez-Aranguren LC, Prada CE, Riaño-Medina CE, Lopez M. Endothelial dysfunction and preeclampsia: role of oxidative stress. Front Physiol 2014;5:372.

15. Girotti MJ, Khan N, Mc Lellan BA. Early measurement of systemic lipid products in plasma of major blunt trauma patients. J Trauma 1991;31:32-5.

16. Gay CA, Gebicki JM. Measurement of protein and lipid hydroperoxides in biological systems by ferric-xylenol orange method. Anal Biochem 2002;30:65-74.

17. Alamdari DH, Paletas K, Pegiou T, Sarigianni M, Befani C, Koliakos G. A novel assay for the evaluation of the prooxidant-antioxidant balance, before and after antioxidant vitamin administration in type II diabetes patients. Clin Biochem 2007;40:248-54.

18. Mankuta D, Elami-Suzin M, Elhayani A, Vinker S. Lipid profile in consecutive pregnancies. Lipids Health Disease 2010;9:58-62.

19. Charlton F, Tooher J, Rye KA, Hennessy A. Cardiovascular risk, lipids and pregnancy: preeclampsia and the risk of later life cardiovascular disease. Heart Lung Circ 2014;23:203-12.

20. Millan J, Pinto X, Munoz A, Zuniga M, Rubies-Prat J, Masani L. Lipoprotein ratios: physiological significance and clinical usefulness in cardiovascular prevention. Vasc Health Risk Manag 2009;5:757-65.
21. Santos-Weiss IC, Rea RR, Fadel-Pichteh CM, Rego FG, Pedrosa F, Gillery P, et al. Picheth G. The plasma logarithm of the triglyceride/ HDL-cholesterol ratio is a predictor of low risk gestational diabetes in early pregnancy. Clin Chim Acta 2013;418:1-4.

22. Jia L, Long S, Fu M, Yan B, Tian Y, Xu Y, et al. Relationship between total cholesterol/high-density lipoprotein cholesterol ratio, triglyceride/high-density lipoprotein cholesterol ratio, and high-density lipoprotein subclasses. Metabolism 2006;55:1141-8.

23. Duhig K, Chappell LC, Shennan AH. Oxidative stress in pregnancy and reproduction. Obstet Med 2016;9:113-6.

24. Cuffe SM, Xu ZC, Perkins AV. Biomarkers of oxidative stress in pregnancy complications. Biomark Med 2017;11:295-306.

25. Samir D, Dalal D, Noura A. Study of oxidative stress during pregnancy. Glob J Pharmaceu Sci 2018;4:555646.

26. Patil SB, Kodliwadmath MV, Kodliwadmath SM. Study of oxidative stress and enzymatic antioxidants in normal pregnancy. Ind J Clin Biochem 2007;2:135-7.

27. Gohil JT, Patel PK, Priyanka G. Evaluation of oxidative stress and antioxidant defence in subject of preeclampsia. Indian J Obstet Gynecol 2011;61:638-40.

28. Rumbold A, Ota E, Hori H, Miyazaki C, Crowther CA. Vitamin E supplementation in pregnancy. Cochrane Database Syst Rev 2015;18:CD004069.

29. Rumbold A, Ota E, Nagata C, Shahrook S, Crowther CA. Vitamin C supplementation in pregnancy. Cochrane Database Syst Rev 2015;18:CD004072.

30. Berti C, Cetin I, Agostoni C, Desoye G, Devlieger R, Emmett PM, et al. Pregnancy and infants' outcome: nutritional and metabolic implications. Crit Rev Food Sci Nutr 2016;56:82-91. 